\title{
Knowledge of human papillomavirus and the human papillomavirus vaccine in European adolescents: a systematic review
}

\author{
Hersha Patel, ${ }^{1}$ Yadava B Jeve, ${ }^{1}$ Susan M Sherman, ${ }^{2}$ Esther L Moss ${ }^{1}$
}

\begin{abstract}
- Additional material is published online only. To view please visit the journal online (http://dx.doi.org/10.1136/ sextrans-2015-052341).

${ }^{1}$ Department of Gynaecology, University Hospitals Leicester, Leicester, UK

${ }^{2}$ School of Psychology, Keele University, Staffordshire, UK
\end{abstract}

\section{Correspondence to}

Dr Hersha Patel, Department of Gynaecology, University Hospitals Leicester, Gwendolen Road, Leicester LE5 4PW, UK; hersha-patel@hotmail.co.uk

Received 26 August 2015 Revised 16 December 2015 Accepted 28 December 2015 Published Online First 20 January 2016

\section{CrossMark}

To cite: Patel $H$, Jeve $Y B$, Sherman SM, et al. Sex

Transm Infect 2016;92:474479.

\begin{abstract}
Background The human papillomavirus (HPV) vaccine is recommended for adolescent girls in many European countries, however there is huge variation in vaccine uptake. Methods A mixed methods systematic review to ascertain the level of HPV and HPV vaccine knowledge that exists among European adolescents. Two electronic databases, Ovid Medline and PsychInfo, were searched from origin to September 2014. Meta-analysis was performed for the two primary outcome measures ('have you heard of HPV?' and 'have you heard of the HPV vaccine?'), assessing for the correlation between gender and knowledge. This was supplemented with meta-synthesis for the remaining associations and secondary outcomes.

Results 18 papers were included in the final review. Overall European adolescents had poor understanding of basic HPV and HPV vaccine knowledge. Meta-analysis identified that female adolescents are more likely to have heard of HPV ( $n=2598 / 5028$ girls versus $n=1033 / 3464$ boys; OR $2.73,95 \% \mathrm{Cl} 1.86-3.99$ ) and the HPV vaccine ( $n=1154 / 2556$ girls versus $n=392 / 2074$ boys; OR 5.64, 95\% Cl 2.43-13.07), compared to males. Age, higher education and a positive vaccination status were also associated with increased awareness. There was limited appreciation of more detailed HPV knowledge and uncertainty existed regarding the level of protection offered by the vaccine and the need for cervical screening post vaccination.
\end{abstract}

Conclusions The delivery of HPV education to European adolescents needs to be re-evaluated, since at present there appears to be significant deficiencies in their basic knowledge and understanding of the subject. Increasing HPV knowledge will empower adolescents to make informed choices regarding participation with HPV related cancer prevention health strategies.

\section{INTRODUCTION}

Human papillomavirus (HPV) is the most common viral infection of the reproductive tract that most men and women who are sexually active will acquire at some point in their lives. ${ }^{1}$ It is the main aetiological factor for cervical cancer with a prevalence of $99.7 \%$ in all cases of cervical cancer. ${ }^{2}$

Following the identification of the role of HPV in the aetiology of cervical cancer, four prophylactic vaccines targeting various high-risk HPV types have been developed. The monovalent vaccine (HPV16), the bivalent vaccine (HPV 16/18), the quadrivalent vaccine (HPV 16/18/6/11) ${ }^{3}$ and more recently, the nonavalent vaccine (HPV 16/18/6/11/ $31 / 33 / 45 / 52 / 58) .{ }^{4}$ The bivalent and quadrivalent vaccines were licensed for use in 2006/2007 and even over this relatively short period the beneficial effects of the vaccine are already evident; with decreases in the incidence of high-grade cervical abnormalities, the prevalence of vaccine HPV types and the incidence of genital warts. ${ }^{4}$

The HPV vaccine is widely recommended in many European countries for female adolescents (aged 918 years ${ }^{5}$ ), however independent of funding discrepancies, the uptake of the vaccine has been variable, ranging from $<5-86 \% .^{6-8}$ Reasons for vaccine uptake are multifactorial, ${ }^{9}{ }^{10}$ however knowledge of HPV and the HPV vaccine have been identified as key predictive factors in increasing uptake. ${ }^{11}$ To date studies have shown that awareness of HPV across all populations is poor. ${ }^{12}{ }^{13} \mathrm{It}$ was hoped that the health promotion campaigns, which preceded vaccine introduction would result in heightened awareness of HPV, thus far the evidence for this is lacking. ${ }^{14}$

HPV vaccination is primarily aimed at adolescents and conveying HPV related health information to this group is arguably the greatest challenge for many healthcare professionals. ${ }^{15}$ In spite of this, it is essential that adolescents fully understand, and are able to critically appraise the information that they are provided, in order for them to make informed choices. ${ }^{16}$

The aim of this systematic review was to assess the level of HPV and HPV vaccine knowledge that exists amongst European adolescents.

\section{METHODS}

A mixed methods systematic review, exploring qualitative and quantitative data, was conducted to assess our aim. The two main primary outcomes that we assessed were: 'have you heard of HPV?' and 'have you heard of the HPV vaccine?'

\section{Search strategy}

We adhered to the PRISMA (Preferred Reporting Items for Systematic Reviews and Meta-Analyses) guidelines for systematic reviews. ${ }^{17}$ Two electronic databases, Ovid Medline and PyscINFO were searched from establishment to September 2014. These databases were used without any limitations and they were deemed to be the most relevant databases for this research question. Searches were conducted using 11 combinations of the keywords 'HPV', 'Papillomavirus', 'Human papilloma virus', 'Health knowledge' 'Attitudes', 'Practice', 'Patient education', 'Vaccine/vaccination' and 'Primary prevention' limited to English language and human participants.

\section{Eligibility criteria}

All studies reporting data specifically on HPV and/ or HPV vaccine knowledge in European 
adolescents were included. The upper age limit, for this review was set at a mean age of 20 years. This was because in some studies, which had been conducted in European secondary schools, the upper age limit reached 20 years. ${ }^{18-21}$ Only studies, which were conducted in a member country of the European Union, were included. We excluded studies in which the main focus was vaccine uptake, acceptability or attitudes. Studies providing insufficient information, for example letters/abstracts only, were also excluded.

\section{Critical appraisal}

Two researchers (HP, ELM) independently reviewed the full texts for quality and suitability. The Critical Appraisal Skills Programme tool was used to review the qualitative studies ${ }^{22}$ and a quality checklist was developed, following the principles identified by Petticrew and Roberts ${ }^{23}$ and using the Centre for Evidence-Based Management tool, ${ }^{24}$ for the quantitative survey studies. Any discrepancies were discussed until a consensus was reached.

\section{Data analysis}

Study features and outcomes were assembled in a tabular form, and formal meta-analysis was performed using RevMan. ${ }^{25} \mathrm{~A}$ random-effects model (using the Mantel-Haenszel (MH) method) was used as the $\mathrm{I}^{2}$ statistic showed heterogeneity of $>50 \%$. The effect estimate was expressed as a pooled OR with 95\% CI and was represented graphically by forest plots. Statistical heterogeneity was examined using the $\chi^{2}$ test and a $p$ value of $<0.05$ was suggestive of heterogeneity. Clinical heterogeneity was examined by assessing the participants, intervention used, study quality and outcome measures. The meta-analysis was supplemented with meta-synthesis for the secondary outcome measures.

\section{RESULTS}

\section{Study characteristics}

The literature search revealed 905 suitable studies following the removal of duplicates (see online supplementary file 1). The full texts of 29 articles that had met the initial inclusion criteria were reviewed and 11 of these studies were excluded. Reasons for exclusion included age of participants, studies not assessing HPV or HPV vaccine knowledge, study assessing the effect of intervention and adequate information not supplied. Studies that had published results in more than one publication were amalgamated resulting in 4 qualitative and 14 quantitative studies to be included in the final review. Publication dates of the studies ranged from 2008 to 2013. Sample size ranged from 217 to 1769 for the quantitative and from 10 to 87 for the qualitative studies. Most of the included studies had been conducted in a Western European country (UK, Italy, Sweden, the Netherlands, Portugal, Germany), with only two from Eastern Europe, both from Hungary. Study characteristics are summarised in web tables 1 and 2 .

\section{Critical appraisal}

The majority of studies were considered to be of moderate to good quality (see online supplementary file 2 ). The response rate was greater than $70 \%$ in all but two studies. ${ }^{20}{ }^{26}$ However many of the studies predominately used convenience sampling and therefore the representativeness of the data may be questioned.

\section{Primary outcomes}

Awareness of HPV

'Have you heard of HPV?'

Twelve of the quantitative studies specifically assessed for this outcome. ${ }^{18-21} 27-34$ Awareness of HPV varied between the studies with the lowest level existing among a cohort of Swedish adolescents, mean age 16 years, where only $5 \%$ had ever heard of HPV $^{18}$ The highest level of awareness of HPV was noted in Italian adolescents, mean age 16 years, where $92 \%$ had heard of HPV. ${ }^{21}$ Awareness of HPV in Italian female adolescents has increased over time, ${ }^{19} 2128$ a similar trend has been noted for male and female adolescents in the Netherlands ${ }^{3134}$ and Sweden. ${ }^{18} 30$

Factors found to be associated with greater HPV awareness included:

1. Vaccinated girls were more likely to have heard of HPV than unvaccinated girls ${ }^{35} \quad(77 \%, \mathrm{n}=683$ vs $8.5 \%, \mathrm{n}=76$; $\mathrm{p}=0.017^{27}$ )in two UK studies, however adolescent boys appeared to have an equal level of awareness as unvaccinated girls in a German study. ${ }^{26}$

2. Higher education in general and, in particular, students studying a medical or health sciences related subject were found to correlate with greater awareness compared with non-health science subjects. ${ }^{31} 3334$

3. Knowledge was found to positively increase with age ${ }^{20}$ and in one Italian study, it was noted that each 1 year increment in age resulted in $14 \%$ increased knowledge. ${ }^{28}$ In addition, a Hungarian study demonstrated that adolescents attending secondary school had better knowledge than those attending primary school $(40 \%, \mathrm{n}=463$ vs $25 \%, \mathrm{n}=152 ; \mathrm{p} \leq 0.001) .{ }^{32}$

4. Only one German study revealed a correlation with sexual experience, showing that students who had previous sexual experience had superior awareness of HPV than those who had never had sex (OR 1.53, 95\% CI 1.01 to 2.12). ${ }^{20}$

5. Gender was found to be the greatest and most consistent predictive factor for having heard of HPV. Meta-analysis of eight quantitative studies showed that adolescent girls are more likely to have heard of HPV compared with the boys, $(\mathrm{n}=2598 / 5028$ girls versus $\mathrm{n}=1033 / 3464$ boys; OR 2.73, 95\% CI 1.86-3.99) (figure 1). ${ }^{19-2130-34}$

\section{Awareness of HPV vaccine}

\section{'Have you heard of the HPV vaccine?'}

Five quantitative studies assessed for this outcome. ${ }^{18} 20213032$ Highest levels of vaccine awareness were again found in Italian adolescents, $94.5 \%$ and $71.3 \%$ of girls and boys, respectively, in the group who had heard of HPV had also heard of the HPV vaccine. ${ }^{21}$ This is a substantial improvement on the findings by Di Giuseppe et $a l^{28}$ who in 2007, found that in Italy only $42 \%$ of those that had heard of cervical cancer, identified HPV vaccination was a preventative measure and only $15 \%$ knew that the vaccine was available in Italy. Knowledge of the HPV vaccine among Swedish adolescents was found to be extremely low, with awareness reaching a maximum of $9.2 \%$ in girls in one study ${ }^{30}$ and in another the overall awareness was only $1.1 \%$ with no significant difference noted in gender. ${ }^{18}$ Lower levels of vaccine awareness corresponded to low awareness of HPV in these cohorts.

Meta-analysis of the four studies that analysed gender differences in awareness, found that girls were more likely to have heard of the HPV vaccine $(n=1154 / 2556$ girls versus $n=392 /$ 2074 boys; OR 5.64, 95\% CI 2.43-13.07) (figure 2) 20213032 and interestingly many adolescent boys felt that it did not even concern them. ${ }^{19}$

\section{Secondary outcomes}

Mode of transmission

On the whole adolescents had a sound understanding of the fact that HPV is a sexually transmitted disease. ${ }^{18} 192126-2830-32343637$ 


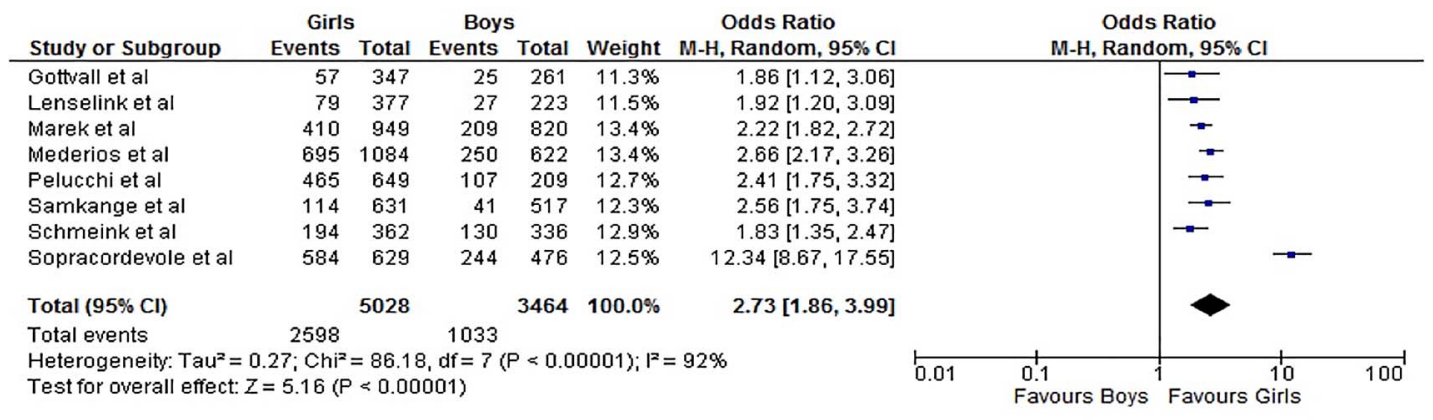

Figure 1 Meta-analysis results for the outcome 'Have you heard of HPV?': A comparison between genders.

First-year medical students in a UK based study, had the greatest level of knowledge regarding mode of $\operatorname{transmission}^{37}$ and the lowest levels were found among groups in which not many had heard of HPV in the first instance. ${ }^{18} 30$ Very few recognised that HPV can be transmitted through genital skin-to-skin contact or non-penetrative intercourse. ${ }^{27} 2832$ Other routes of transmission, which had been erroneously suggested included, the airborne route where students had applied the knowledge that they held for other viruses, such as the influenza virus, to HPV. ${ }^{38}$ In addition some mentioned blood transfusions, ${ }^{33}$ the use of public toilets and poor intimate hygiene. $^{21}$

\section{Link to cervical cancer and genital warts}

The correlation between high-risk HPV and cervical cancer was poorly understood, with a group of 12-13-year-old girls in the UK believing that HPV and cervical cancer were the same. ${ }^{38}$ In two UK based studies, despite the majority of the participants having heard of HPV, only $50 \%$ or less were aware of its link to cervical cancer ${ }^{27} 37$ and one of these studies had been conducted in 1st-year medical students. ${ }^{37}$

Compared with boys, girls in general ${ }^{20} 21303234$ and vaccinated girls ${ }^{26}$ were found to have greater knowledge of the relationship between HPV and cervical cancer. Misconceptions existed regarding the hereditary nature of cervical cancer, 76\% of medical faculty students incorrectly stated hereditary cause as a risk factor. $^{31}$

Understanding of the association between HPV and genital warts was low, ${ }^{18} 21273032$ with the greatest levels existing in 15-19 year-old Hungarian boys. ${ }^{32}$

Level of protection offered by the vaccine

Adolescents appreciated that the HPV vaccine provided protection against cervical cancer but uncertainty existed on the exact level of protection offered. ${ }^{27} 3637$ Only a few knew that it was still possible to get cervical cancer after receiving the vaccine $^{27}$ and that the vaccine only offers protection against some of the high-risk HPV subtypes. ${ }^{37}$ One group of girls thought that the vaccine would not stop them from getting cervical cancer but that it would stop them dying from it. ${ }^{36}$ A minority mentioned that it may also protect against 'STDs'19 and genital warts. ${ }^{21}$ Others falsely believed that the HPV vaccine also provided protection against HIV/ AIDs and viral hepatitis. ${ }^{21}$ The two Swedish studies revealed that adolescents felt their intention to use condoms would decrease after receiving the HPV vaccine and that other adolescents would feel the same, ${ }^{18}{ }^{30}$ however Italian adolescents acknowledged the need to use condoms during sexual activity following vaccination. ${ }^{21}$

Need for cervical screening in the future

Four $^{27} 363839$ UK based studies found that the need for cervical screening following vaccination was not clear. Some thought that cervical screening was being provided as a 'backup' for those who were still concerned after being vaccinated rather than it being mandatory for all. ${ }^{39}$ Vaccinated female medical students in the UK and Italian adolescents appeared to be more aware of the requirement for cervical screening. ${ }^{21} 37$

\section{DISCUSSION}

Our review reveals that the level of HPV and HPV vaccine knowledge among European adolescents remains suboptimal. These results reinforce the findings of another review of European adolescents which found that of all the sexually transmitted diseases the lowest level of awareness existed for HPV. ${ }^{40}$ Recent systematic reviews concluded that increased HPV knowledge does result in increased vaccine uptake and acceptability. ${ }^{11} 12$ When vaccine recipients and their parents are provided with balanced information, they have improved knowledge and risk perception, which results in increased vaccine uptake. ${ }^{41}$

In this review we attempted to identify factors, which may be associated with greater HPV knowledge. The following factors were explored looking for any correlations.

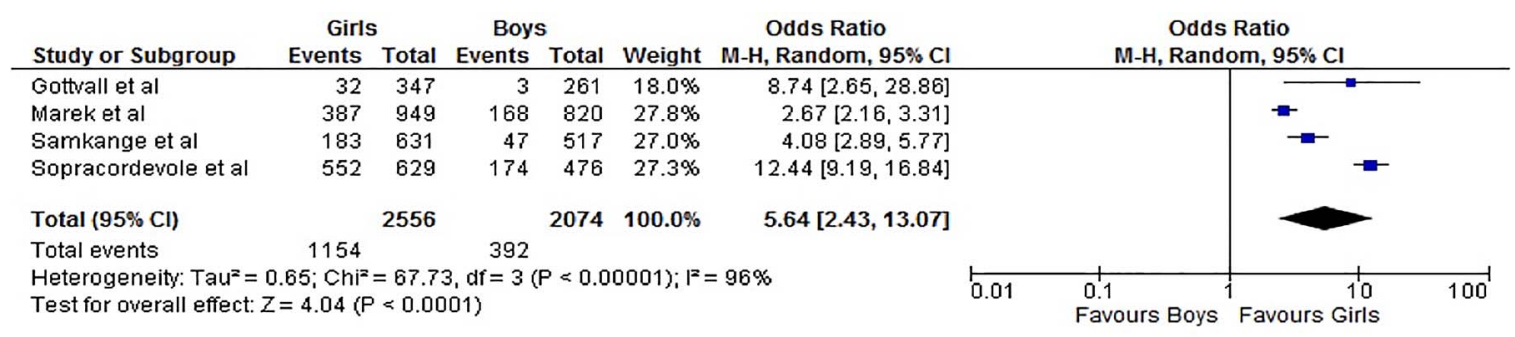

Figure 2 Meta-analysis results for the outcome 'Have you heard of the HPV vaccine?': A comparison between genders. 


\section{Gender}

An emergent theme from our review was that female gender correlated with increased knowledge. Studies from across Europe showed that female adolescents had more knowledge than boys, a trend which is also replicated in the adult population. ${ }^{13}$ This is likely to be reflective of the fact that HPV is primarily thought of as a women's health problem and that the majority of countries presently only recommend the HPV vaccine for women. However HPV is responsible for malignancies in men too, including cancers of the anus, penis, oral cavity and oropharynx ${ }^{42}$ and furthermore men share an equal burden for HPV related genital warts. ${ }^{43}$ Currently HPV vaccination for adolescent boys is only recommend by a few, including America, Canada, Australia and Austria. Furthermore, the UK's Joint Committee on Vaccination and Immunisation has recently recommended that men who have sex with men (aged 1640 years) should also be offered the HPV vaccine. ${ }^{44}$ The costeffectiveness of the vaccine has been debated as the rational against vaccinating adolescent boys. ${ }^{44}$ Moreover it has been argued that boys will receive a degree of protection from the herd immunity effect of vaccinated girls. However HPV vaccination is only part of the solution, abstaining from high-risk behaviour is equally important. Parents feel that by including boys in HPV education and in the vaccination campaign we can reinforce their responsibility in sexual health ${ }^{45}$ and avoid girls feeling stigmatised by being the sole target. ${ }^{46}$ The challenge will be to develop educational materials which are equally relevant to both genders.

\section{Introduction of the HPV vaccine into national immunisation programmes and funding}

The timing of HPV vaccine introduction across Europe has varied and integration into national immunisation programmes and/or funding are not universal. The results of this review do not consistently support the concept that the availability of a fully state-funded HPV immunisation programme at the time of the study resulted in increased awareness. The highest levels of HPV awareness were noted in Italian adolescents, in a study conducted in 2009, 1-2 years after the HPV vaccine was introduced into the Italian national vaccination schedule, ${ }^{47}$ and the lowest levels were seen in Sweden in 2007, 3 years prior to implementation of the HPV vaccine. ${ }^{48}$ However, studies from Hungary $^{32}$ and the Netherlands ${ }^{31} 34$ showed a greater level of HPV awareness than Germany, ${ }^{20}$ despite the former studies being conducted prior to the implementation of a free national HPV vaccination programme in their respective countries ${ }^{5}$ and the German study taking place 4 years post 'free of cost' vaccine introduction in Germany. ${ }^{49}$ These findings imply that much more complex factors are involved in acquiring knowledge and that the introduction of national HPV vaccination alone is not predictive.

\section{Organisation of a vaccination programme, school based versus primary care/private}

It has been proposed that countries, such as the UK and Australia, which have school based vaccination programmes have achieved higher vaccination coverage rates. ${ }^{50}$ However the studies within our review showed that the UK, which operates a school based vaccination programme, has a vaccine coverage rate of $86 \%{ }^{7}$ but Portugal, where the HPV vaccine is administered in primary care and health centres, ${ }^{49}$ also had a comparable coverage rate of $84 \% .^{6}$ The organisation of HPV vaccine administration did not correlate with level of knowledge in this review, however knowledge was related to vaccine uptake: Italy, UK and Portugal had the highest levels of vaccine uptake and also the greatest amount of knowledge.

\section{Delivery of information}

Vaccine introduction in many European countries was preceded by health promotion campaigns, including school based presentations. $^{5152}$ The successfulness of these campaigns may be questioned, as overall HPV knowledge in this cohort remains insufficient. This raises the possibility that information may have been inappropriately targeted in some countries. McCusker et al, who specifically compared HPV knowledge, pre and post a HPV health promotion campaign in the UK, found that the campaign did not over time add much in terms of HPV knowledge. $^{37}$

Adolescents in this review described their main sources of information as media, schools and the internet. ${ }^{28} 303237$ They felt that although written information was useful, it needed to be more user-friendly and complemented with nurse-led small group discussions. ${ }^{38}$ Currently a recommendation cannot be made on the most effective way of communicating HPV related information, as no one method has been found to be superior. ${ }^{50}$ However it is apparent that adolescents are requesting more information than that which is presently available. ${ }^{28} \quad 3032 \quad 3536$ In addition the challenge will be maintaining knowledge over time. ${ }^{53}$ When delayed posteducation follow-up has been conducted, even after only 1 month, the initially noted benefit is diminished. ${ }^{54}$ This suggests that there is a need for continued educational reinforcement as opposed to a single intervention session. This is particularly important with regards to cervical screening post vaccination.

\section{Strengths and limitations}

There have been previous systematic reviews assessing HPV knowledge in various different populations ${ }^{12-145556}$ but this is the most up-to-date review that focuses on the primary target group for the HPV vaccine: adolescents.

Our review was a comprehensive mixed methods review, using this approach provides more accurate data, which is likely to have greater impact. ${ }^{57}$

However it was limited by the scope of the primary studies. Five of the survey studies had not validated their questionnaires and there were considerable variations in the way specific acceptance of HPV knowledge were assessed, for example prompted versus open questions. In our review an upper age limit of 20 years was adopted, this meant that some of the included studies had been conducted at university level and although the average age did not exceed 20 years, this is a vastly different population. Lastly, the study populations were all heterogeneous and therefore difficult to truly compare.

\section{Conclusions}

Current evidence suggests that European adolescents have limited understanding of basic factual HPV knowledge. HPV plays a pivotal role in the present and future of preventative cancer strategies for both genders. Methods of delivering HPV information to this complex group need to be re-evaluated to promote increased awareness and empower them to make informed choices regarding future preventative health behaviours. 


\section{Key messages}

- Current evidence suggests that European adolescents lack basic factual human papillomavirus (HPV) knowledge and that female gender is consistently associated with greater HPV awareness.

- HPV is becoming more prevalent and is responsible for malignancies in both genders, however the risk of acquiring it can be reduced.

- Previous evidence has shown that increased HPV awareness does result in increased HPV vaccine uptake.

- In order to increase participation with HPV related cancer prevention health strategies, methods of delivering HPV related information need to be re-evaluated.

\section{Handling editor Jackie A Cassell}

Contributors HP, YBJ, SMS and ELM were involved in planning the review and contributed equally to manuscript preparation and review. HP conducted the literature search and data extraction, performed the meta synthesis of the qualitative data and wrote the initial draft of the manuscript. YBJ performed the statistical analysis for the meta analysis. HP and ELM contributed to quality assessment of the studies. All authors approved the final report.

Competing interests SMS has been awarded the following grant; 2015-North Staffordshire Medical Institute, Pump Priming Award. Mothers and Daughters: Exploring knowledge about and attitudes towards cervical screening and HPV vaccination.

Provenance and peer review Not commissioned; externally peer reviewed.

Data sharing statement All authors had full access to all the data in the study.

\section{REFERENCES}

1 WHO. World Health Organisation, Human papillomavirus (HPV) and cervical cancer. Secondary World Health Organisation, Human papillomavirus (HPV) and cervical cancer 2015. http://www.who.int/mediacentre/factsheets/fs380/en/

2 Walboomers JM, Jacobs MV, Manos MM, et al. Human papillomavirus is a necessary cause of invasive cervical cancer worldwide. J Pathol 1999;189:12-19.

3 Lowndes CM. Vaccines for cervical cancer. Epidemiol Infect 2006;134:1-12.

4 Joura $E A$, Giuliano $A R$, Iversen $O E$, et al. A 9-valent HPV vaccine against infection and intraepithelial neoplasia in women. N Eng/ J Med 2015;372:711-23.

5 European Centre for Disease Prevention and Control (ECDC). Vaccine ScheduleRecommended immunisations for human papillomavirus infection. Secondary European Centre for Disease Prevention and Control-Vaccine ScheduleRecommended immunisations for human papillomavirus infection. http:/l vaccine-schedule.ecdc.europa.eu/Pages/Scheduler.aspx

6 European Centre for Disease Prevention and Control (ECDC). Introduction of HPV vaccines in European Union countries-an update. 2012.

7 PHE. Public Health England: Human Papillomavirus (HPV) Vaccine Coverage in England, 2008/09 to 2013/14.A review of the full six years of the three-dose schedule. https://www.gov.uk/government/uploads/system/uploads/attachment_data/ file/412264/HPV_Vaccine_Coverage_in_England_200809_to_201314.pdf

8 Poljak M, Seme K, Maver PJ, et al. Human papillomavirus prevalence and type-distribution, cervical cancer screening practices and current status of vaccination implementation in central and Eastern Europe. Vaccine 2013;31(Suppl 7):H59-70.

9 Canfell $K$, Egger $S$, Velentzis $L S$, et al. Factors related to vaccine uptake by young adult women in the catch-up phase of the National HPV Vaccination Program in Australia: results from an observational study. Vaccine 2015;33:2387-94.

10 Fisher $\mathrm{H}$, Trotter $\mathrm{CL}$, Audrey $\mathrm{S}$, et al. Inequalities in the uptake of human papillomavirus vaccination: a systematic review and meta-analysis. Int I Epidemiol 2013:42:896-908.

11 Kessels SJ, Marshall HS, Watson M, et al. Factors associated with HPV vaccine uptake in teenage girls: a systematic review. Vaccine 2012;30:3546-56.

12 Chan ZCY, Chan TS, Ng KK, et al. A systematic review of literature about women's knowledge and attitudes toward Human Papillomavirus (HPV) Vaccination. Public Health Nurs 2012;29:481-9.

13 Klug SJ, Hukelmann M, Blettner M. Knowledge about infection with human papillomavirus: a systematic review. Prev Med 2008;46:87-98.

14 Hendry M, Lewis R, Clements A, et al. "HPV? Never heard of it!": a systematic review of girls' and parents' information needs, views and preferences about human papillomavirus vaccination. Vaccine 2013;31:5152-67.
15 Brown EC, Little P, Leydon GM. Communication challenges of HPV vaccination. Fam Pract 2010;27:224-9.

16 General Medical Council (GMC). Consent: patients and doctors making decisions together. 2008.

17 Moher D, Liberati A, Tetzlaff J, et al. Preferred reporting items for systematic reviews and meta-analyses: the PRISMA statement. Ann Intern Med 2009;151:264-9.

18 Höglund AT, Tydén T, Hannerfors AK, et al. Knowledge of human papillomavirus and attitudes to vaccination among Swedish high school students. Int I STD AIDS 2009;20:102-7.

19 Pelucchi C, Esposito S, Galeone C, et al. Knowledge of human papillomavirus infection and its prevention among adolescents and parents in the greater Milan area, Northern Italy. BMC Public Health 2010;10:378.

20 Samkange-Zeeb F, Mikolajczyk RT, Zeeb H. Awareness and knowledge of sexually transmitted diseases among secondary school students in two German cities. J Community Health 2013;38:293-300.

21 Sopracordevole F, Cigolot F, Gardonio V, et al. Teenagers' knowledge about HPV infection and HPV vaccination in the first year of the public vaccination programme. Eur J Clin Microbiol Infect Dis 2012;31:2319-25.

22 CASP. CRITICAL APPRAISAL SKILLS PROGRAMME-10 questions to help you make sense of qualitative research. Secondary CRITICAL APPRAISAL SKILLS PROGRAMME -10 questions to help you make sense of qualitative research 2011. http://www. casp-uk.net/\#!casp-tools-checklists/c18f8

23 Petticrew M, Roberts H. Systematic reviews in the social sciences: a practical guide. Oxford: Blackwell, 2006.

24 (CEBMa) CFE-BM. Critical Appraisal of a Survey. Secondary Critical Appraisal of a Survey. http://www.cebma.org/wp-content/uploads/ Critical-Appraisal-Questions-for-a-Survey.pdf

25 Review Manager 5.3. The Cochrane Collaboration. Copenhagen: The Nordic Cochrane Centre, 2014[ program].

26 Stöcker $\mathrm{P}$, Dehnert M, Schuster M, et al. Human papillomavirus vaccine uptake, knowledge and attitude among 10th grade students in Berlin, Germany, 2010. Hum Vaccin Immunother 2013;9:74-82.

27 Bowyer HL, Marlow LA, Hibbitts S, et al. Knowledge and awareness of HPV and the HPV vaccine among young women in the first routinely vaccinated cohort in England. Vaccine 2013;31:1051-6.

28 Di Giuseppe G, Abbate R, Liguori G, et al. Human papillomavirus and vaccination: knowledge, attitudes, and behavioural intention in adolescents and young women in Italy. Br J Cancer 2008;99:225-9.

29 Forster AS, Marlow LA, Wardle J, et al. Interest in having HPV vaccination among adolescent boys in England. Vaccine 2012;30:4505-10.

30 Gottvall M, Larsson M, Höglund AT, et al. High HPV vaccine acceptance despite low awareness among Swedish upper secondary school students. Eur I Contracept Reprod Health Care 2009;14:399-405.

31 Lenselink CH, Schmeink CE, Melchers WJ, et al. Young adults and acceptance of the human papillomavirus vaccine. Public Health 2008;122:1295-301.

32 Marek E, Dergez T, Rebek-Nagy G, et al. Adolescents' awareness of HPV infections and attitudes towards HPV vaccination 3 years following the introduction of the HPV vaccine in Hungary. Vaccine 2011;29:8591-8.

33 Medeiros R, Ramada D. Knowledge differences between male and female university students about human papillomavirus (HPV) and cervical cancer: implications for health strategies and vaccination. Vaccine 2010;29:153-60.

34 Schmeink CE, Gosens KC, Melchers WJ, et al. Young adults awareness of HPV and vaccine acceptance after introduction of the HPV vaccine in the Dutch national vaccination program. Eur I Gynaecol Oncol 2011;32:481-6.

35 Williams K, Forster A, Marlow L, et al. Attitudes towards human papillomavirus vaccination: a qualitative study of vaccinated and unvaccinated girls aged 1718 years. J Fam Plann Reprod Health Care 2011;37:22-5.

36 Hilton S, Smith E. "I thought cancer was one of those random things. I didn't know cancer could be caught...": adolescent girls' understandings and experiences of the HPV programme in the UK. Vaccine 2011;29:4409-15.

37 McCusker SM, Macqueen I, Lough G, et al. Gaps in detailed knowledge of human papillomavirus (HPV) and the HPV vaccine among medical students in Scotland. BMC Public Health 2013;13:264.

38 Racktoo S, Coverdale G. 'HPV? Never heard of it' students and the HPV vaccine. Br J School Nurs 2009;4:328-34.

39 Henderson L, Clements A, Damery S, et al. 'A false sense of security'? Understanding the role of the HPV vaccine on future cervical screening behaviour: a qualitative study of UK parents and girls of vaccination age. J Med Screen 2011;18:41-5.

40 Samkange-Zeeb FN, Spallek L, Zeeb H. Awareness and knowledge of sexually transmitted diseases (STDs) among school-going adolescents in Europe: a systematic review of published literature. BMC Public Health 2011;11:727.

41 Wegwarth 0 , Kurzenhäuser-Carstens S, Gigerenzer G. Overcoming the knowledge-behavior gap: the effect of evidence-based HPV vaccination leaflets on understanding, intention, and actual vaccination decision. Vaccine 2014;32:1388-93.

42 Dillner J. Prevention of human papillomavirus-associated cancers. Semin Oncol 2015;42:272-83. 
43 Stanley M. Perspective: vaccinate boys too. Nature 2012;488:S10.

44 Kirby T. UK committee recommends HPV vaccination for MSM. Lancet Oncol 2015;16:e7.

45 Noakes K, Yarwood J, Salisbury D. Parental response to the introduction of a vaccine against human papilloma virus. Hum Vaccin 2006;2:243-8.

46 Katz ML, Reiter PL, Heaner S, et al. Acceptance of the HPV vaccine among women, parents, community leaders, and healthcare providers in Ohio Appalachia. Vaccine 2009;27:3945-52.

47 King LA, Levy-Bruhl D, O'Flanagan D, et al. Introduction of human papillomavirus (HPV) vaccination into national immunisation schedules in Europe: results of the VENICE 2007 survey. Euro Surveill 2008;13:pii: 18954.

48 Tegnell A, Dillner J, Andrae B. Introduction of human papillomavirus (HPV) vaccination in Sweden. Euro Surveill 2009;14:pii: 19119.

49 Markowitz LE, Tsu V, Deeks SL, et al. Human papillomavirus vaccine introductionthe first five years. Vaccine 2012;30(Suppl 5):F139-48.

50 Fu LY, Bonhomme LA, Cooper SC, et al. Educational interventions to increase HPV vaccination acceptance: a systematic review. Vaccine 2014;32:1901-20.

51 Steckelberg A, Albrecht M, Kezle A, et al. Impact of numerical information on risk knowledge regarding human papillomavirus (HPV) vaccination among schoolgirls: a randomised controlled trial. Ger Med Sci 2013;11:Doc15.
52 Chadenier GM, Colzani E, Faccini M, et al. Assessment of the first HPV vaccination campaign in two northern Italian health districts. Vaccine 2011;29:4405-8.

53 Brabin L, Stretch R, Roberts SA, et al. Survey of girls' recall of a film providing information on human papillomavirus and cervical cancer 6 months after an offer of vaccination. Vaccine 2010;28:4210-14.

54 Doherty K, Low KG. The effects of a web-based intervention on college students' knowledge of human papillomavirus and attitudes toward vaccination. Int I Sex Health 2008;20:223-32.

55 Marlow LAV. HPV vaccination among ethnic minorities in the UK: knowledge, acceptability and attitudes. Br J Cancer 2011;105:486-92.

56 Mishra A. Implementing HPV vaccines: public knowledge, attitudes, and the need for education. Int Q Community Health Educ 2010;31:71-98.

57 Harden A. Mixed-Methods Systematic Reviews: Integrating Quantitative and Qualitative Findings. Focus: A Publica- tion of the National Center for the Dissemination of Disability Research (NCDDR) Technical Brief. Secondary Mixed-Methods Systematic Reviews: Integrating Quantitative and Qualitative Findings. Focus: A Publica- tion of the National Center for the Dissemination of Disability Research (NCDDR) Technical Brief 2010. http://www.ktdrr.org/ktlibrary/ articles_pubs/ncddrwork/focus/focus25/Focus25.pdf 